\title{
DECONVOLUTION OF NEUTRON DEPTH PROFILING SPECTRA
}

\author{
John T. MAKI ${ }^{1,2)}$, Ronald F. FLEMING ${ }^{1)}$, and Dietrich H. VINCENT ${ }^{2)}$ \\ 1) Center for Analytical Chemistry, National Bureau of Standards, Gaithersburg, Maryland 20899, USA \\ 2) Department of Nuclear Engineering, University of Michigan, Ann Arbor, Michigan 48109-2100, USA
}

Received 2 December 1985 and in revised form 28 March 1986

Iterative methods for determining deconvoluted depth profiles from measured neutron depth profiling (NDP) energy spectra are presented. The methods account for energy broadening caused by system noise, energy straggling, multiple small angle scattering, and geometrical acceptance angles. Examples of the methods are given for known and unknown profiles of helium-3 and nitrogen-14.

\section{Introduction}

Neutron depth profiling (NDP) is successfully being used to determine concentration profiles of selected nuclides in various materials [1]. The technique makes use of a limited number of thermal neutron induced charged particle reactions. Table 1 lists the reactions with cross sections greater than one barn which are suitable for NDP measurements. Measurements are performed by illuminating a sample with a beam of thermal neutrons. When parent nuclides absorb a neutron, they emit a charged particle and a recoil atom. Since the incident neutron is of low energy (about $0.02 \mathrm{eV}$ ), particle emission is isotropic and the initial kinetic energies of the particles are uniquely defined by the reaction $Q$ value. As the particles travel through the host material, they lose energy in a nearly continuous manner. A solid state detector measures the residual energy of the particles that have emerged from the surface of the sample and have entered the sensitive volume of the detector. This information is stored as an energy spectrum and ideally correlates to a depth profile by the relations

$x=\int_{E(x)}^{E_{0}} \mathrm{~d} E / S(E), \quad$ and

$d=x \cos (\theta)$,

where $E_{0}$ is the initial energy of the particle, $E(x)$ is the detected residual energy, $S(E)$ is the particle stopping power in the host material, $x$ is the pathlength of the particle, $\theta$ is the emittance angle relative to the sample normal, and $d$ is the depth within the sample of the parent nuclide.

As a result of energy broadening, particles originating from a depth $d$ are not detected with a discrete energy $E(x)$, as indicated by eq. (1), but give a distribution of energies defined by the measurement response function. The response function is the energy distribution of the initially monoenergic particles emitted from a given depth. The broadening is caused by a) electronic noise in the detection system, b) energy straggling from the statistical nature of the slowing down process, c) multiple small angle scattering, which results in variations of particle pathlengths, and d) geometrical acceptance angles, which result in variations of detectable particle pathlengths.

Depth profiles are determined from energy spectra by deconvolution, or the unfolding of the response function from the measured data. Three main types of deconvolution procedures have been developed to perform this task [2]. These procedures include the direct method, the Fourier transform method, and the iterative method. The direct method suffers from noise in the deconvolute and is sensitive to the form of the original data. The Fourier transform method is prone to introducing mathematical artifacts into the deconvolute. To avoid these problems, the iterative method was selected for deconvolution development. This paper will describe

Table 1

Suitable nuclides for NDP measurements with reactions, reaction $Q$ values, and thermal neutron cross sections.

\begin{tabular}{llcc}
\hline Element & Reaction & $\begin{array}{l}\text { Cross } \\
\text { section } \\
(\mathrm{b})\end{array}$ & $\begin{array}{l}\text { Reaction } \\
Q \text { Value } \\
\text { (keV) }\end{array}$ \\
\hline $\mathrm{He}$ & $\left.{ }^{3} \mathrm{He}(\mathrm{n}, \mathrm{p})\right)^{3} \mathrm{H}$ & 5333 & 764 \\
$\mathrm{Li}$ & ${ }^{6} \mathrm{Li}(\mathrm{n}, \alpha)^{3} \mathrm{H}$ & 940 & 4782 \\
$\mathrm{Be}$ & ${ }^{7} \mathrm{Be}(\mathrm{n}, \mathrm{p})^{7} \mathrm{Li}$ & 48000 & 1644 \\
$\mathrm{~B}$ & ${ }^{10} \mathrm{~B}(\mathrm{n}, \alpha)^{7} \mathrm{Li}$ & 3837 & 2790 \\
$\mathrm{~N}$ & ${ }^{14} \mathrm{~N}(\mathrm{n}, \mathrm{p})^{14} \mathrm{C}$ & 1.83 & 626 \\
$\mathrm{Na}$ & ${ }^{22} \mathrm{Na}(\mathrm{n}, \mathrm{p})^{22} \mathrm{Ne}$ & 31000 & 3624 \\
$\mathrm{~K}$ & ${ }^{40} \mathrm{~K}(\mathrm{n}, \mathrm{p})^{40} \mathrm{Ar}$ & 4.4 & 2287 \\
$\mathrm{Ni}$ & ${ }^{59} \mathrm{Ni}(\mathrm{n}, \alpha)^{56} \mathrm{Fe}$ & 12.3 & 5097 \\
\hline
\end{tabular}


two iterative methods, the chi-square minimization [3] and the Van Cittert [4] procedures for deconvoluting NDP measurements performed at the National Bureau of Standards Reactor. A description of the NBS/NDP facility is presented elsewhere [5].

\section{Method}

A depth profile is related to measured data by the convolution integral

$Y\left(x^{\prime}\right)=\int_{0}^{\infty} \mathrm{d} x C(x) f\left(x^{\prime}, x\right)$

where $Y(x)$ is the measured distribution at depth $x$, $C(x)$ is the actual concentration at depth $x$, and $f\left(x^{\prime}, x\right)$ is the response function. Since NDP spectra are stored in a histogram manner as a function of energy by a multichannel analyzer, and the maximum depth to which a profile can be measured is related to the range $R$ of the emitted particle, eq. (3) is rewritten with a change of variables as

$Y_{n}=\int_{E_{n}-\Delta}^{E_{n}+\Delta} \mathrm{d} E^{\prime} \int_{0}^{R} \mathrm{~d} x C(x) F\left(E^{\prime}, E(x)\right)$

or as

$Y_{n}=\int_{0}^{R} \mathrm{~d} x C(x) \int_{E_{n}-\Delta}^{E_{n}+\Delta} \mathrm{d} E^{\prime} F\left(E^{\prime}, E(x)\right)$,

where $Y_{n}$ is the measured counts in channel $n, E_{n}$ is the mean energy of channel $n, \Delta$ is one half the energy width of a channel, $E(x)$ is the energy corresponding to a particle emitted from depth $x$, and $F\left(E^{\prime}, E(\mathrm{x})\right)$ is the energy response function. The energy response function is assumed to be a Gaussian of the form

$F\left(E^{\prime}, E(x)\right)=\frac{1}{\sqrt{2 \pi} \sigma_{E(x)}} \exp \left[\frac{-1}{2}\left(\frac{E^{\prime}-E(x)}{\sigma_{E(x)}}\right)^{2}\right]$

where $\sigma_{E(x)}$ is one standard deviation of the Gaussian. The standard deviation of the response function is determined by the magnitude of the various mechanisms of energy broadening mentioned above. These mechanisms can be treated separately and the standard deviations of each of the four contributions are added in quadrature to form $\sigma_{E(x)}$. Thus,

$\sigma_{E(x)}=\left[\sum_{i}\left(\sigma_{E(x)}\right)_{i}^{2}\right]^{1 / 2}$

The integral of the energy response function is expressed in terms of the error function whereby eq. (5) may be written as

$$
\begin{aligned}
Y_{n}=\frac{1}{2} \int_{0}^{R} \mathrm{~d} x C(x)[ & \operatorname{erf}\left(\frac{E_{n}+\Delta-E(x)}{\sqrt{2} \sigma_{E(x)}}\right) \\
& \left.-\operatorname{erf}\left(\frac{E_{n}-\Delta-E(x)}{\sqrt{2} \sigma_{E(x)}}\right)\right] .
\end{aligned}
$$

Implicit in eq. (7) is the assumption that each mechanism of energy broadening may be represented by a Gaussian. The magnitude of each component is then described by its associated standard deviation. The evaluation of these energy broadening mechanisms follows.

\subsection{Contributions to energy broadening}

\subsubsection{System noise}

Detection system noise arises from a) electronic noise of the amplifier chain, b) intrinsic noise of the detector, primarily resulting from leakage current, and c) the statistical variation of ionizing events produced within the detector by a particle. The system noise is then characterized as

$\sigma_{\mathrm{SN}}^{2}=\sigma_{\mathrm{ELECT}}^{2}+\sigma_{\mathrm{DET}}^{2}+\sigma_{\mathrm{ION}}^{2}$

where the subscripts of the standard deviations are $\mathrm{SN}$ for the total system noise, ELECT for the electronic noise, DET for the intrinsic detector noise, and ION for the ionization event variation.

Electronic noise and the detector intrinsic noise can be measured by feeding a signal from a precision pulser through the detection system. The resulting standard deviation of the pulser signal, $\sigma_{\text {PULSER }}$, is then expressed as

$\sigma_{\text {PULSER }}^{2}=\sigma_{\text {ELECT }}^{2}+\sigma_{\text {DET }}^{2}$.

This contribution to the system noise is observed to be independent of pulse amplitude and hence, particle energy.

For heavy charged particles, some energy is expended in phonon production near the end of their tracks within a detector [6]. The fraction of energy diverted from producing ionizing events is a function of the initial energy and mass of the particle. Since the pulse amplitude produced by a solid state detector is directly proportional to the number of ionizing events produced within its volume, phonon production results in a variation of pulse heights (and hence, energy determination) for heavy particles of the same initial energy.

For electrons or hydrogen isotopes (such as protons or tritons) variations resulting from phonon production are essentially zero and $\sigma_{\mathrm{rON}}=0$ [7]. Therefore, for light particles, the detection system noise is a constant and is represented as

$\sigma_{\mathrm{SN}}^{2}=\sigma_{\mathrm{PULSER}}^{2}$ 


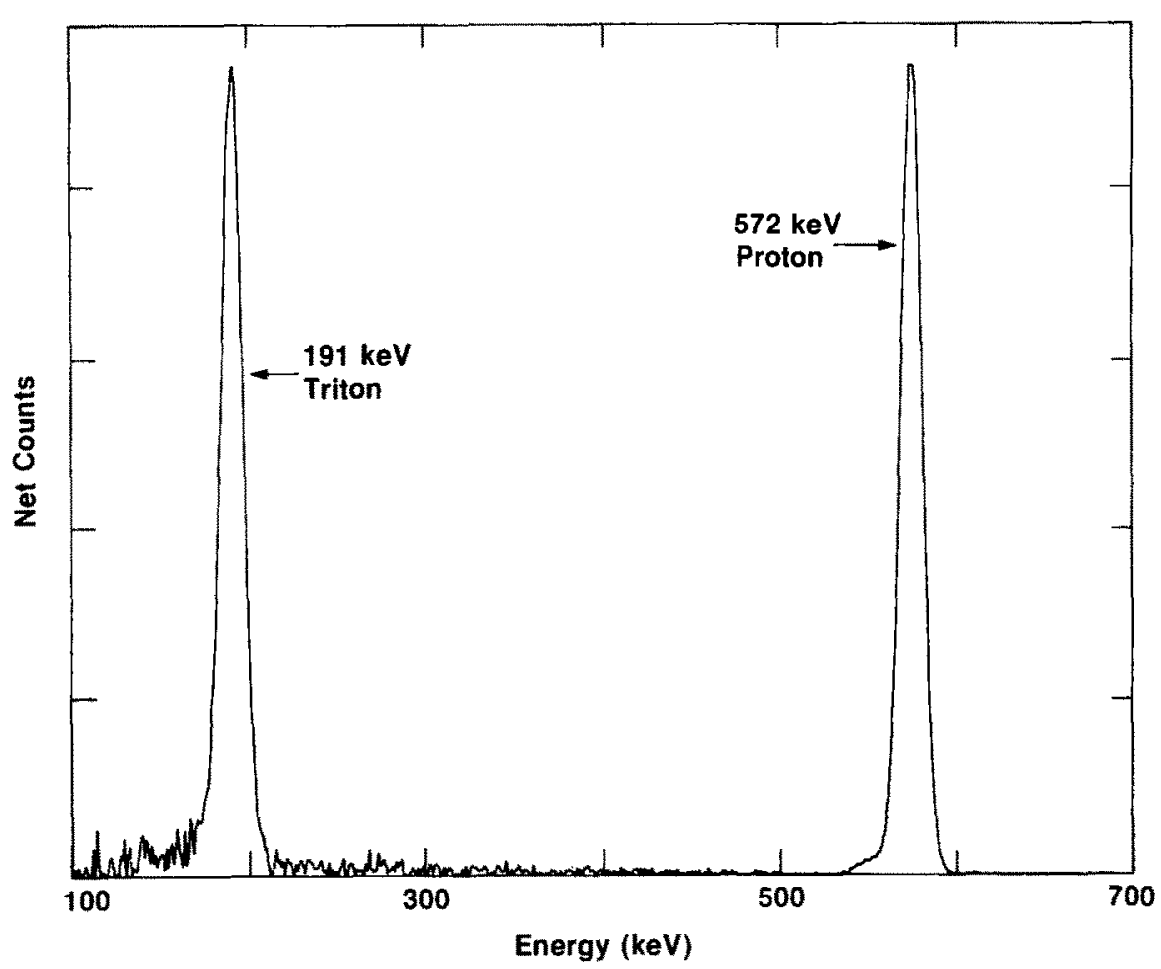

Fig. 1. NDP cnergy spcctrum of the ${ }^{3} \mathrm{He}(\mathrm{n}, \mathrm{p})^{3} \mathrm{H}$ reaction from a $250 \mathrm{eV}$ helium-3 surface deposition on aluminum.

For heavier particles (with atomic numbers greater than or equal to two) where $\sigma_{I O N}$ is not negligible, the system noise is a function of particle energy.

The fact that system noise is independent of energy for light particles can be verified by a NDP measurement of a surface layer. Since particles emitted from a surface layer do not travel through bulk material, measured peak widths are due only to system noise. Results from a NDP measurement of a surface layer of helium-3 deposited on an aluminum foil are presented in fig. 1 . From the kinematics of the ${ }^{3} \mathrm{He}(n, p){ }^{3} \mathrm{H}$ reaction, the emitted $572 \mathrm{keV}$ protons and the $191 \mathrm{keV}$ tritons have equal probability of being detected which is shown by the equal area under each peak. With equal peak areas and peak widths, this measurement indicates that system noise is independent of energy for light particles.

Energy dependent system noise for heavy particles can be determined by NDP measurements of selected surface layers at particle energies defined by the reaction $Q$ value. Due to the limited number of nuclides suitable for NDP measurements, only a few surface layer determinations of system noise for a given particle are possible. This would necessitate extrapolation to obtain system noise values at other energies.

\subsubsection{Energy straggling}

The contribution to energy broadening by energy straggling is determined by the Bohr model [8]. The magnitude of this contribution is determined from

$\sigma_{\text {STRAGG }}^{2}=4 \pi Z_{1}^{2} Z_{2} e^{4} N x$,

where $\sigma_{\text {STRAGG }}$ is the standard deviation of broadening caused by energy straggling, $Z_{1}$ is the atomic number of the emitted particle, $Z_{2}$ is the atomic number of the host material, $e$ is the electronic charge, $N$ is the atomic density of the host material, and $x$ is the pathlength of the emitted particle through the host material.

It has been shown that the Bohr model well represents the straggling data for protons in nickel when the proton energy loss is less than $350 \mathrm{keV}$ regardless of initial proton energy [9]. Eq. (1) has been evaluated for the case of helium- 3 in nickel and is presented in fig. 2 with the proton and triton residual energies plotted as a function of pathlength. As evident from the figure, the maximum depth that can be profiled is about $3 \mu \mathrm{m}$ and for distributions deeper than $2 \mu \mathrm{m}$, the proton and triton signals would overlap. Therefore, NDP measurements of helium and nitrogen with profiles deeper than about $2 \mu \mathrm{m}$ are generally not encountered. For these cases, proton energy loss is less than $350 \mathrm{keV}$ and the Bohr straggling model is adequate for NDP deconvolution. However, for profiles deeper than $2 \mu \mathrm{m}$, another expression for energy straggling would be required.

\subsubsection{Multiple small angle scattering}

Energy broadening caused by multiple small angle 


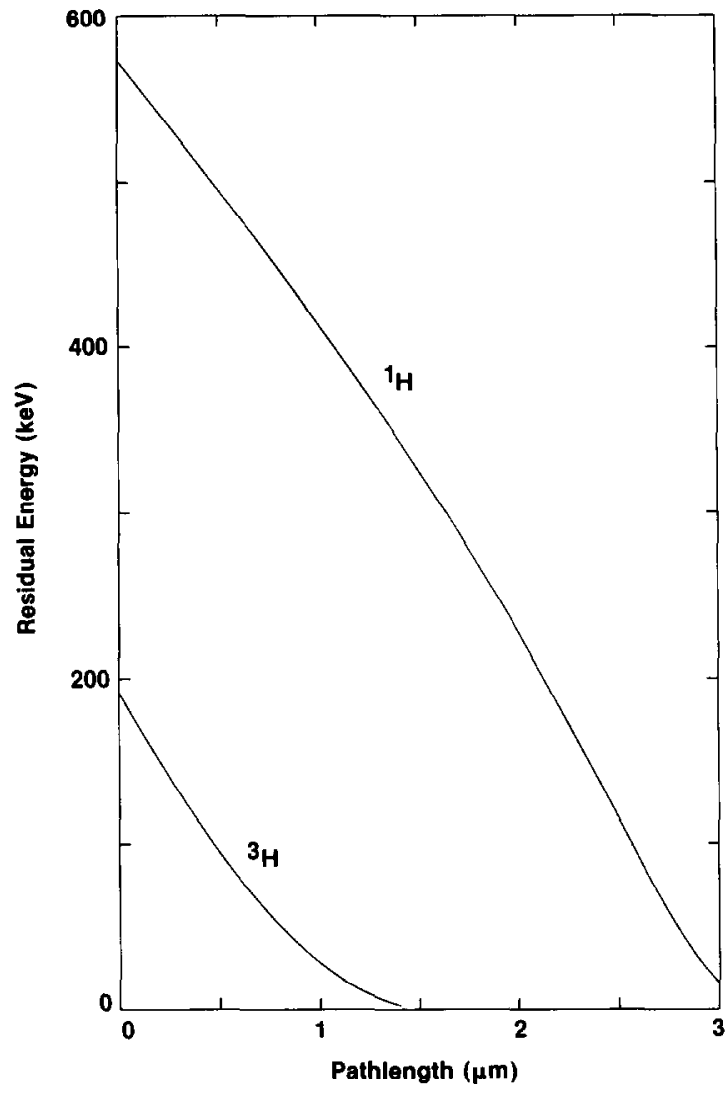

Fig. 2. Residual energies of protons and tritons from the ${ }^{3} \mathrm{He}(\mathrm{n}, \mathrm{p}){ }^{3} \mathrm{H}$ reaction in nickel.

scattering may be approximated from

$\sigma_{\text {SCATT }}=S(E(x)) \sigma_{x \text {-SCATT }}$,

where $\sigma_{\text {SCATT }}$ is the standard deviation of broadening caused by scattering, $S(E(\mathrm{x}))$ is the particle stopping power, and $\sigma_{x \text {-SCATT }}$ is the spread in particle pathlengths due to scattering. The error propagation formula is applied to

$x=d / \cos \theta$

to determine the particle pathlength spread from a spread of emittance angles due to scattering, $\sigma_{\theta_{s}}$, which is expressed as

$\sigma_{x-\mathrm{SCATT}}=d\left(\frac{\sin \theta}{\cos ^{2} \theta}\right) \sigma_{\theta_{s}}$.

Substituting the average value of the emittance angle $\bar{\theta}$ for $\theta$, and a deflection angle width $\sigma_{\psi}$, for $\sigma_{\theta_{s}}$ in eq. (15), eq. (13) may be written as

$\sigma_{\mathrm{SCATT}}=S(E(x)) d\left(\frac{\sin \bar{\theta}}{\cos ^{2} \bar{\theta}}\right) \sigma_{\psi}$.
The deflection angle width may be expressed as $[10,11]$

$\sigma_{\psi}=\frac{2 Z_{1} Z_{2} e^{2}}{\bar{E} a} \frac{2}{2.355} C\left(\pi a^{2} N x\right)^{M}$,

where $a$ is the screening parameter defined as $0.885 a_{0}$ $\left(Z_{1}^{2 / 3}+Z_{2}^{2 / 3}\right)^{-1 / 2}, a_{0}$ is the Bohr radius equal to 5.29 $\times 10^{-11} \mathrm{~m}, \bar{E}$ is the average residual energy of the particle, $C$ and $M$ are fitting parameters, and the other variables are as defined above. Data from Spahn and Groeneveld [12] was used to determine the fitting parameters giving $C=0.30$ and $M=0.85$.

\subsubsection{Geometrical acceptance angles}

Due to the finite solid angle subtended between the sample and detector, a range of emittance angles and hence, pathlengths, exists for a given depth of particle emission. Variation in pathlengths results in a corresponding variation in detected particle residual energy. The contribution to energy broadening by geometrical pathlength broadening, $\sigma_{\mathrm{GEOM}}$, is approximated by

$\sigma_{\mathrm{GEOM}}=S(E(x)) \sigma_{x-\mathrm{GEOM}}$,

where $\sigma_{x-G E O M}$ is the spread in particle pathlengths due to the possible range of acceptance angles. The spread in pathlengths is determined from

$\sigma_{x-\mathrm{GEOM}}^{2}=\int_{0}^{\pi / 2} P(\theta)(x-\bar{x})^{2} \mathrm{~d} \theta$,

where $P(\theta)$ is a normalized probability distribution of detectable emission angles. Since

$x=d / \cos \theta$

then

$\bar{x}=\int_{0}^{\pi / 2} P(\theta) \frac{d}{\cos \theta} \mathrm{d} \theta$

or

$\bar{x}=d\left\langle\frac{1}{\cos \theta}\right\rangle$.

Substituting eqs. (20) and (22) into eq. (19) the result is $\sigma_{x-G E O M}^{2}=d^{2} \int_{0}^{\pi / 2} P(\theta)\left[\frac{1}{\cos \theta}-\left\langle\frac{1}{\cos \theta}\right\rangle\right]^{2} \mathrm{~d} \theta$.

The normalized detection probability distribution is expressed as

$P(\theta)=\frac{W(\theta)}{\int_{0}^{\pi / 2} W(\theta) \mathrm{d} \theta}$,

where $W(\theta)$ is the weighting function given by

$W(\theta)=\int_{0}^{R_{\mathrm{s}}} I\left(r_{\mathrm{s}}\right) \Delta \psi\left(r_{\mathrm{s}}, \theta\right) \mathrm{d} r_{\mathrm{s}}$.

Here, $R_{\mathrm{s}}$ is the radius of the sample, and $I\left(r_{\mathrm{s}}\right)$ is the normalized neutron intensity incident on the sample at a given sample radius $r_{\mathrm{s}}$. Defined further in a following 
paragraph, $\Delta \psi\left(r_{s}, \theta\right)$ is the "detection coverage" for a given emittance angle and sample radius.

The neutron beam used for NDP measurements at NBS is circular in cross section with a maximum, uniform intensity diameter of $9.5 \mathrm{~mm}$. Beyond this diameter, the intensity falls off linearly with a full width at half maximum of $13 \mathrm{~mm}$. Since a sample may be positioned at any angle $\xi$, relative to the neutron beam axis, the sample will see an ellipsoidal neutron intensity distribution. For a given sample radius, $r_{s}$, and independent of sample circumferential angle $\phi$, an equivalent or average neutron beam radius $\bar{R}_{\mathrm{nb}}$, is determined as

$\bar{R}_{\mathrm{nb}}=\frac{r_{s}}{\pi / 2} \int_{0}^{\pi / 2}\left[\cos ^{2} \xi \cos ^{2} \phi+\sin ^{2} \phi\right]^{1 / 2} \mathrm{~d} \phi$,

from which a normalized neutron intensity $I\left(r_{\mathrm{s}}\right)$ is determined from the actual beam map using $\bar{R}_{\mathrm{nb}}$.

For on-axis geometry as shown in fig. 3 where the sample and detector faces are parallel, and the sample normal from its center intersects the detector center, a detector coverage value $\Delta \psi\left(r_{\mathrm{s}}, \theta\right)$ may be determined from

$R_{\mathrm{d}}^{2}=S^{2}+r_{\mathrm{s}}^{2}-2 r_{\mathrm{s}} S \cos (\Delta \psi / 2)$

with

$S=D \tan \theta$.

Here, $R_{\mathrm{d}}$ is the detector radius, $D$ is the sample to detector distance, $\theta$ is the emittance angle, $S$ is the radius of the emittance cone in the plane of the detector, $r_{\mathrm{s}}$ is a given sample radius, and $\Delta \psi$ is the maximum angle to which the emittance cone intersects the

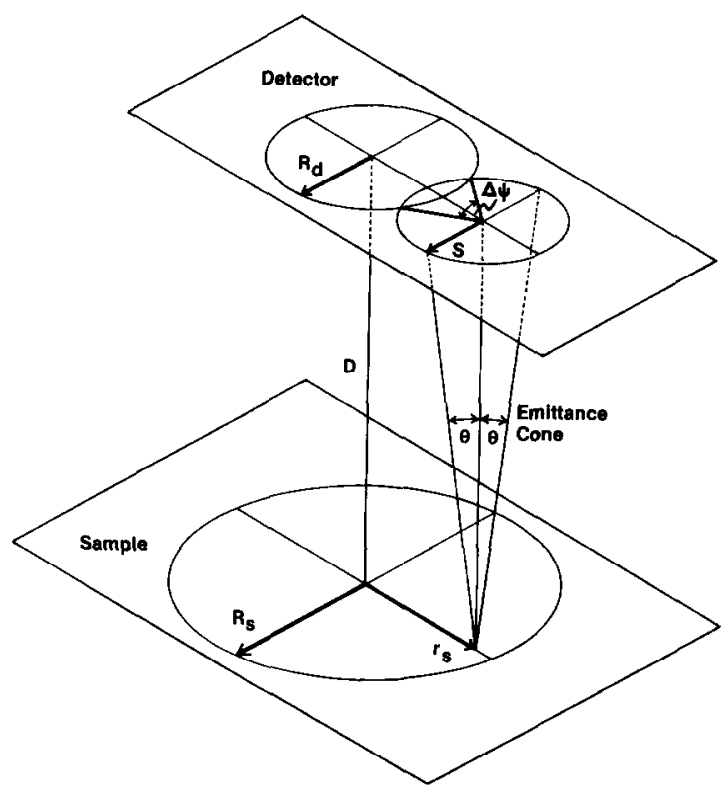

Fig. 3. Geometry used for NDP measurements. detector. Eq. (27) is solved for $\Delta \psi$ and expressed as

$\Delta \psi\left(r_{s}, \theta\right)=2 \arccos \left[\frac{S^{2}+r_{\mathrm{s}}^{2}-R_{\mathrm{d}}^{2}}{2 r_{\mathrm{s}} S}\right]$.

When the arc cos argument is less than -1 , the entire emittance cone intersects the detector, and $\Delta \psi=2 \pi$. When the arc cos argument is greater than 1 , the emittance cone does not intersect the detector and $\Delta \psi=0$.

Summarizing, with the detection coverage expressed by eq. (29) and the normalized neutron intensity determined by eq. (26) and the neutron beam map, the weighting function expressed by eq. (25) is numerically evaluated for a given emittance angle. The weighting function evaluations are then used to determine numerically the normalized detection probability distribution expressed by eq. (24) which is used to determine $\sigma_{x \text {-GEOM }}$ given by eq. (23). Once $\sigma_{x \text {-GEOM }}$ is determined, the geometrical acceptance angle contribution to energy broadening, $\sigma_{\mathrm{GEOM}}$ is calculated according to eq. (18).

A normalized detection probability distribution as calculated by eq. (24) is presented in fig. 4. Also displayed in fig. 4 are the results of a Monte Carlo calculation of the probability distribution. The Monte Carlo distribution contains $1.5 \times 10^{6}$ successful trials and verifies the validity of the numerical calculation.

An example evaluation of each of the four contributions to energy broadening is presented in fig. 5 for protons emitted by helium-3 in nickel. Note that the

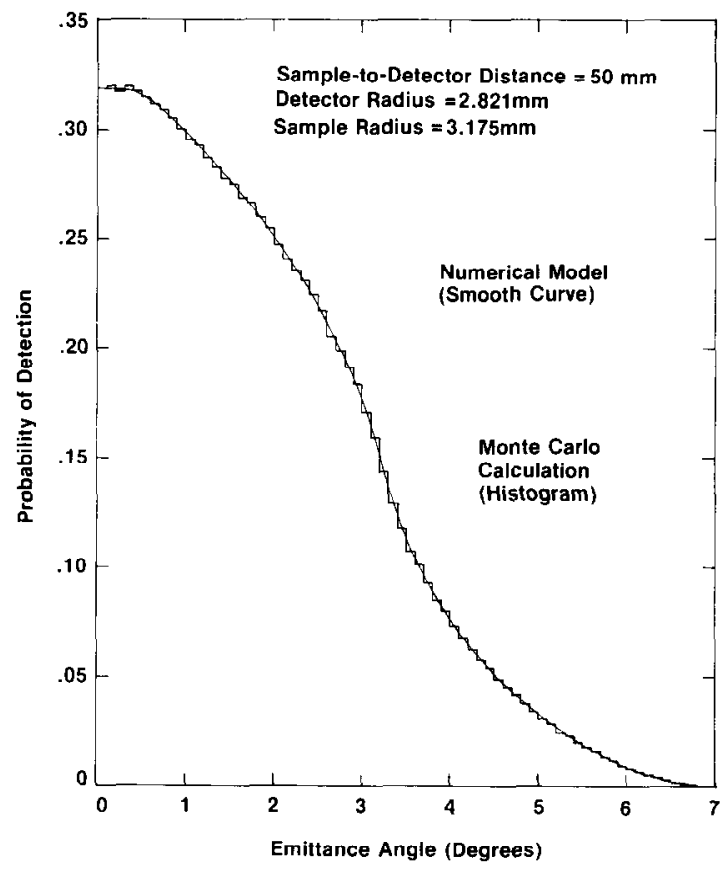

Fig. 4. Normalized detection probability distribution for on-axis sample-detector geometry. 


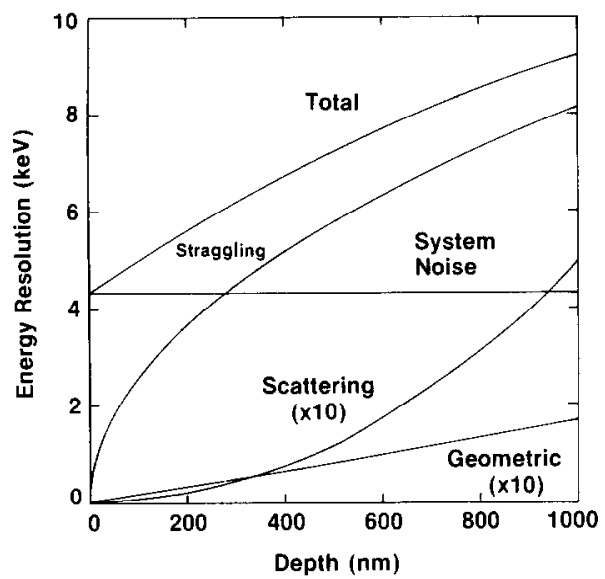

Fig. 5. NDP energy resolution for helium-3 in nickel.

total energy broadening is determined primarily by system noise and energy straggling.

\subsection{Depth profile deconvolution}

\subsubsection{Chi-square minimization method}

The solution of the convolution integral, eq. (8), for a depth profile by the chi-square minimization method is as follows. The form of the depth profile is assumed and its function is placed within the integrand. For a given set of parameters describing the function (such as the mean and standard deviation for a Gaussian profile), the integral is then numerically evaluated by the adaptive incremental Simpson's method [13] for each channel of the measured spectrum using stopping power values tabulated by Ziegler [14]. This distribution is normalized to the same area (total counts) as the measured spectrum and represents the convoluted profile. The convoluted profile should simulate the measured spectrum whereby a goodness of fit is indicated by a chi-square value. The chi-square value is determined as

$\chi^{2}=\frac{1}{N-P} \sum\left(\frac{Y_{n}-Y_{n}^{\prime}}{\sigma_{Y_{n}}}\right)^{2}$,

where $N$ is the number of channels in the spectrum, $P$ is the number of parameters describing the depth profile function, $Y_{n}$ is the measured counts in channel $n$ with a Poisson counting uncertainty of $\sigma_{Y_{n}}$, and $Y_{n}^{\prime}$ is the calculated counts in channel $n$. If the chi-square value has not been minimized, a different set of parameters describing the depth profile function is chosen and the above evaluation is repeated. When chi-square has been minimized, the assumed profile function is normalized to the area of the measured spectrum and thus represents the deconvoluted depth profile. Uncertainties associated with the final parameters describing the de- convoluted profile are then calculated according to the graphical formalism of Rogers [15].

\subsubsection{Van Cittert deconvolution method}

Another technique to determine depth profiles from measured spectra is the iterative Van Cittert deconvolution method. This method does not require trial and error assumptions of the depth profile function. However, the method does require that both the measured and deconvoluted profiles be smooth and slowly varying. When eq. (8) is represented as

$Y_{n}=\sum_{n} C_{n} G\left(E_{n}, E(x)\right)$,

where $C_{n}$ is the number of counts in channel $n$ representing the deconvoluted profile, and $G\left(E_{n}, E(x)\right)$ is the integral of the energy response function, the Van Cittert method evaluates the deconvoluted profile after $i$ iterations as

$C_{n_{i}}=C_{n_{i-1}}+Y_{n}-\sum_{n} C_{n_{i-1}} G\left(E_{n}, E(x)\right)$.

The first iteration uses $C_{n_{0}}=Y_{\mathrm{n}}$. After several iterations, the corrections to the $i$ th evaluation consist mostly of noise. Therefore, after the last iteration, the noise is smoothed [16] by evaluating

$C_{n}=C_{n_{i}}-i\left[Y_{n}-\sum_{n} C_{n_{i}} G\left(E_{n}, E(x)\right)\right]$,

which then represents the deconvoluted depth profile.

\section{Results}

The chi-square minimization method was used to deconvolute the proton peak of the surface helium-3 layer on aluminum presented in fig. 1. A delta function at the surface of the sample was assumed and is represented by the broken line in fig. 6 . This function was convoluted and is displayed as the solid curve in the figure with the measured data represented by solid circles. The assumed delta distribution is not displayed to the same scale as the convoluted spectrum which is normalized to the same area as the measured spectrum. The convoluted distribution closely matches the measured data over most of the spectrum. The measured data indicates that helium may have diffused into the aluminum and become trapped below the surface.

A NDP measurement of a silicon nitride layer on silicon with a silicon dioxide overcoat [17] is presented in fig. 7. The proton peak from the ${ }^{14} \mathrm{~N}(n, p){ }^{14} \mathrm{C}$ reaction was deconvoluted by the chi-square minimization method employing a square wave function for the silicon nitride layer. The resulting deconvoluted depth profile, which is displayed by broken lines, indicates that the silicon nitride layer is $80 \pm 1 \mathrm{~nm}$ thick and that 


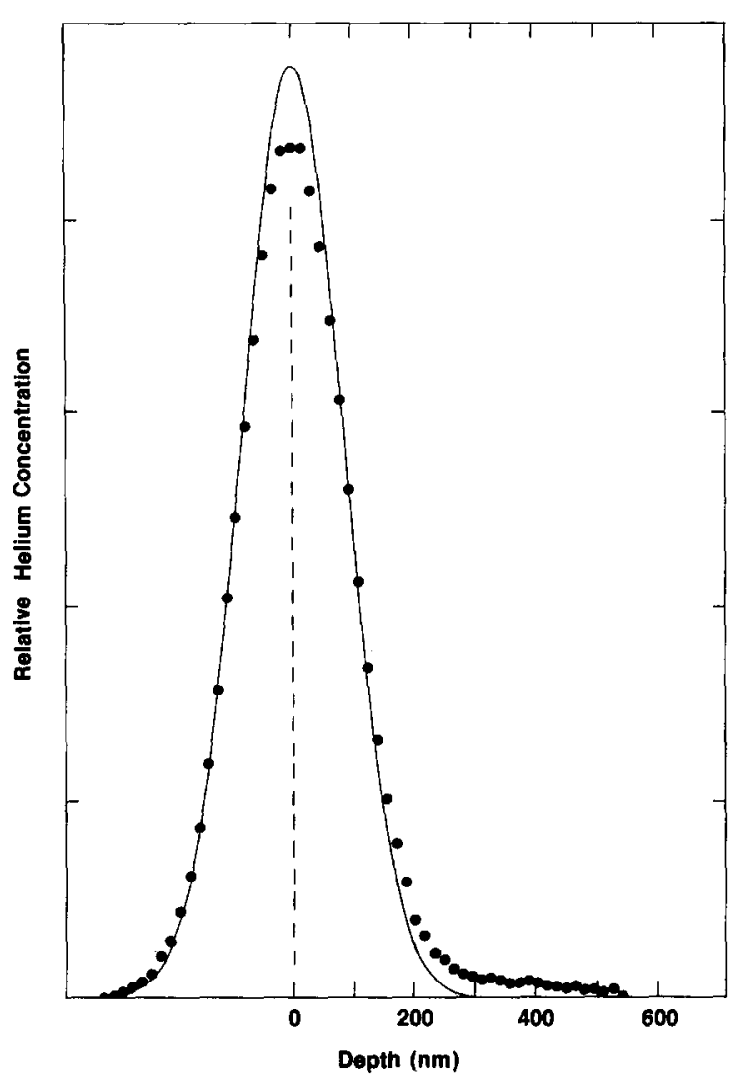

Fig. 6. NDP depth profile of a helium-3 surface layer on aluminum depicting the deconvoluted profile $(---)$, convoluted profile $(-)$, and the measured data $(\bullet)$.

the overcoat is $459 \pm 1 \mathrm{~nm}$ thick. A Rutherford backscattering (RBS) measurement performed on the same sample determined a silicon nitride thickness of 80 $\mathrm{nm}$ with a $470 \mathrm{~nm}$ overcoat.

The results from a NDP measurement of a nitrogen implant at $90 \mathrm{keV}$ to a dose of $5 \times 10^{21}$ at. $\mathrm{m}^{-2}$ in a tungsten carbide-cobalt alloy [18] is presented in fig. 8 . Again, the chi-square minimization method was used to deconvolute the spectrum. The resulting deconvoluted depth profile is represented as a truncated Gaussian with a mean depth of $53 \pm 2 \mathrm{~nm}$ and a standard deviation of $49 \pm 2 \mathrm{~nm}$. An expected result of deconvolution is that the deconvoluted profile, which is normalized to the same area as the measured distribution, displays a more sharply peaked distribution than the measured data.

Fig. 9 presents the results of a NDP measurement for a $150 \mathrm{keV}$ helium-3 implant in single crystal nickel to a dose of $2 \times 10^{20}$ at. $\mathbf{m}^{-2}$. A Pearson IV distribution [19] was employed using the chi-square minimization method for deconvolution. The deconvoluted depth profile is characterized by a mean depth of $365.5 \pm 0.1$ $\mathrm{nm}$, a standard deviation of $112.2 \pm 0.2 \mathrm{~nm}$, a skewness

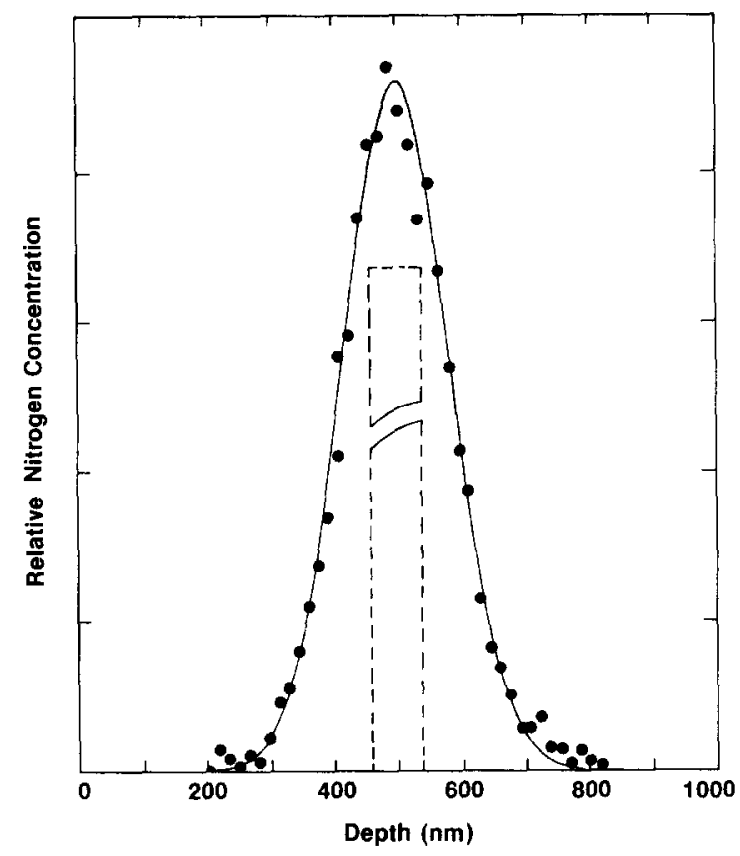

Fig. 7. NDP depth profile of a $80 \mathrm{~nm}$ layer of silicon nitride on silicon with a $460 \mathrm{~nm}$ overcoat of silicon dioxide. Profile legend as for fig. 6 .

of $-2.075 \pm 0.002$, and a kurtosis of $26.94 \pm 0.05$. The deconvoluted depth profile is also presented in fig. 10 with the results from the Winterbon four moment computer code [20], and the Monte Carlo TRIM code [21]. The moments for each of the above three profiles are

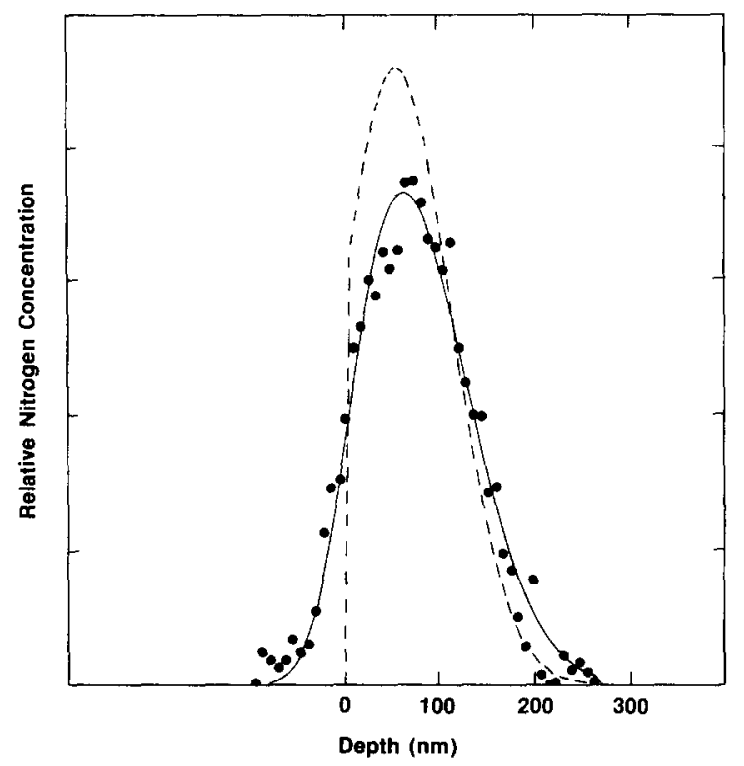

Fig. 8. NDP depth profile of a $90 \mathrm{keV}$ nitrogen-14 implant in tungsten carbide-cobalt to a dose of $5 \times 10^{21}$ at. $\mathrm{m}^{-2}$. Profile legend as for fig. 6 . 


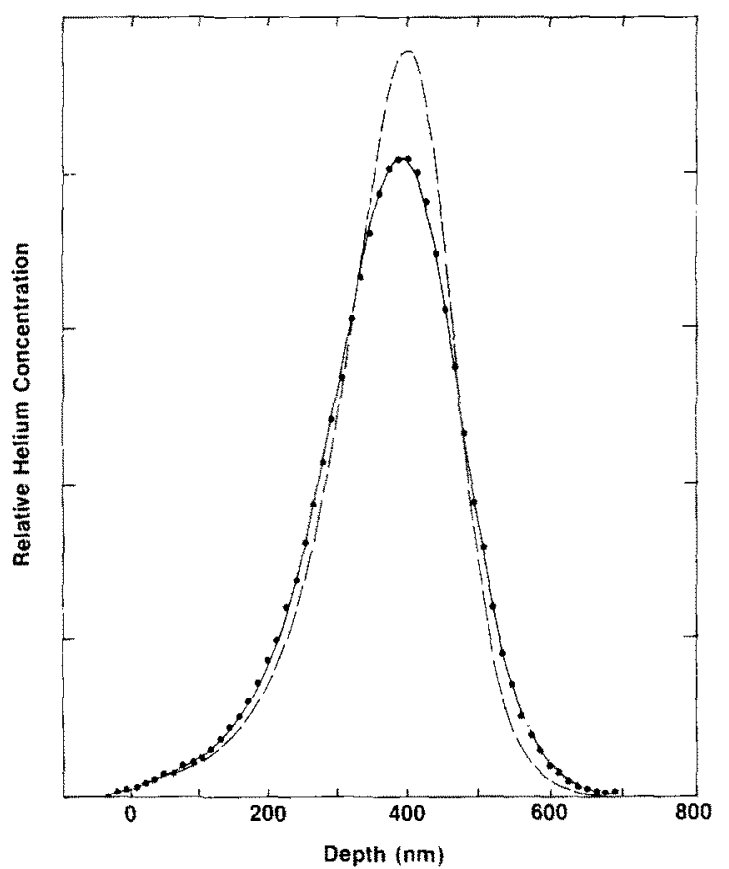

Fig. 9. NDP depth profile of a $150 \mathrm{keV}$ helium-3 implant in single crystal nickel to a dose of $2 \times 10^{20}$ at. $\mathrm{m}^{-2}$. Profile legend as for fig. 6 .

listed in table 2. The discrepancies between the deconvoluted NDP measurement and the predicted profiles maybe due to the lack of sufficiently accurate stopping power evaluations in the computer codes, and possible channeling effects in the single crystal nickel sample which are not accounted for by the computer codes.

Deconvolution of the $150 \mathrm{keV}$ helium-3 implant discussed above was also performed by the Van Cittert

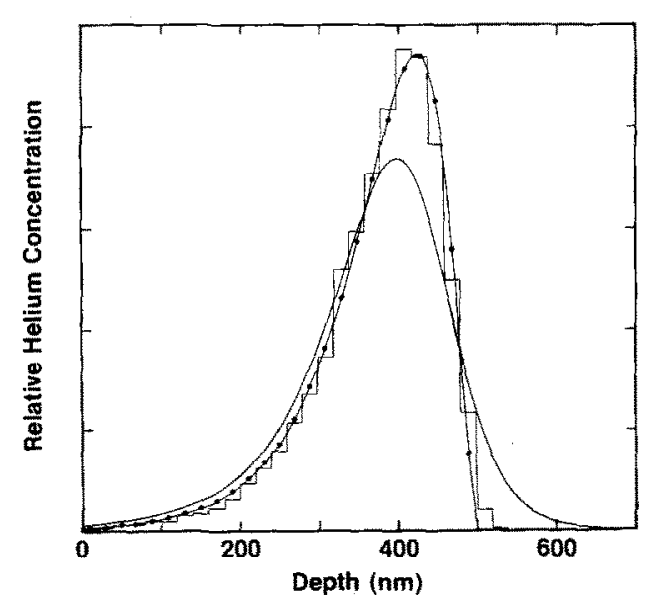

Fig. 10. Depth profiles for a $150 \mathrm{keV}$ helium-3 implant in nickel obtained by NDP deconvolution (- - ), Winterbon computer program (-), and by the Monte Carlo TRIM code (histogram).

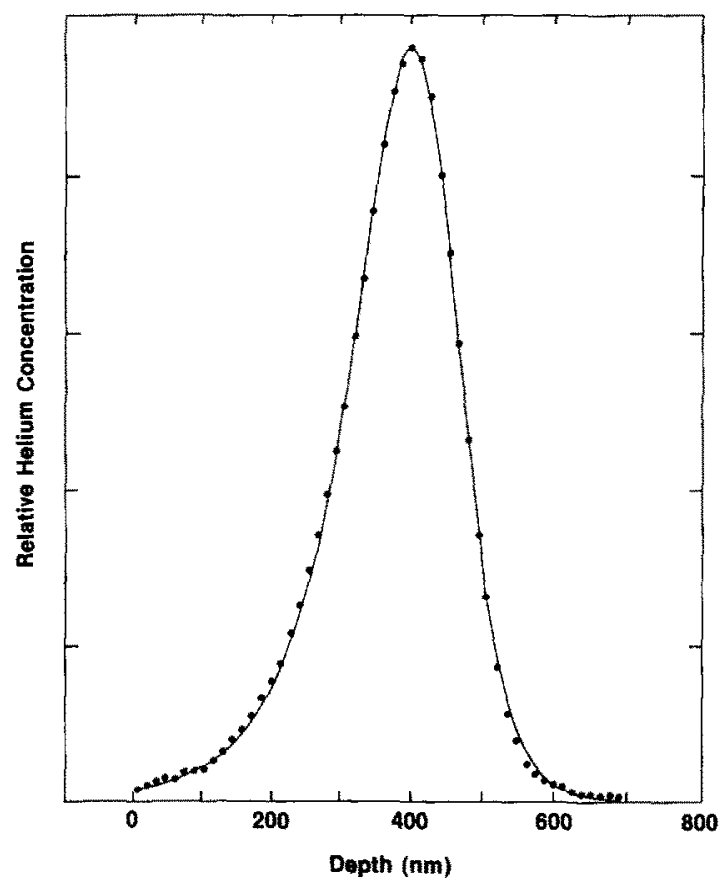

Fig. 11. Comparison of deconvoluted depth profiles for the 150 $\mathrm{keV}$ helium-3 implant in nickel data presented in fig. $9 \mathrm{ob}-$ tained by the Van Cittert method (e), and by the chi-square minimization method (-

Table 2

Comparison of depth profile moments for a $150 \mathrm{keV}$ helium-3 implant in nickel.

\begin{tabular}{lllll}
\hline Method & $\begin{array}{l}\text { Mean } \\
(\mathrm{nm})\end{array}$ & $\begin{array}{l}\text { Standard } \\
\text { deviation } \\
(\mathrm{nm})\end{array}$ & $\begin{array}{l}\text { Skew- } \\
\text { ness }\end{array}$ & Kurtosis \\
\hline Deconvoluted & & & & \\
$\quad$ NDP measurement & 366 & 112 & -2.08 & 26.9 \\
Winterbon code & 371 & 86 & -1.62 & 7.70 \\
TRIM code & 375 & 83 & -1.58 & 8.15 \\
\hline
\end{tabular}

method. The resulting deconvoluted depth profile is presented in fig. 11. The Van Cittert calculation was performed with seven iterations and closely matches the result of the chi-square minimization method which is also displayed in the figure.

\section{Discussion}

The Van Cittert method of deconvolution is limited to slowly varying distributions. For example, delta or square wave depth profiles cannot be treated due to their infinite slopes at the sides of the distribution. Also, the Van Cittert method requires good counting statistics in the original measured data else nonphysical perturba- 
tions are introduced into the resulting deconvoluted profile. For these reasons the helium-3 surface layer and the nitrogen meaurements could not be deconvoluted by this method.

The chi-square minimization method of deconvolution can be used with any distribution and has no requirements pertaining to counting statistics. However, this method, unlike the Van Cittert method, requires apriori knowledge of the depth profile. For example, ion implants in metals are widely known to be best modeled by a four moment distribution. Therefore, the $150 \mathrm{keV}$ helium-3 implant in nickel data was deconvoluted assuming a Pearson IV distribution. The NDP measurement technique has high sensitivity to helium-3 and the resulting high precision counting statistics enabled the four moments to be unambiguously extracted from the data. NDP has less sensitivity to nitrogen than helium because the reaction cross section is a factor of 3000 smaller. Thus, the poorer counting statistics of the nitrogen implant data in tungsten carbide-cobalt did not justify deconvolution with a Pearson IV distribution. The nitrogen implant data was, however, adequately modeled by a truncated Gaussian distribution.

It has been demonstrated that NDP spectra can be deconvoluted by the iterative, chi-square minimization method. With suitable distributions, deconvolution can also be performed by the Van Cittert method.

The authors wish to thank R. Gregory Downing of the National Bureau of Standards for his assistance in the maintenance and operation of the NDP facility and for his construction of the stopping power data files. Appreciation goes to Kenneth S. Grabowski and Phillip R. Malmberg of the Naval Research Laboratory for performing the helium-3 implants in nickel. Finally, the authors wish to thank John H. Albers of the National Bureau of Standards for performing the TRIM code and Winterbon computer program calculations.

\section{References}

[1] R.G. Downing, J.T. Maki, and R.F. Fleming, Microelectronics Processing: Inorganic Materials Characterization,
ACS Symposium Series 295, ed., L.A. Casper (American Chemical Society, Washington, DC., 1986) ch. 9.

[2] A.F. Carley and R.W. Joyner, J. Electron Spectros. Relat. Phenom. 16 (1979) 1.

[3] S. Hoffman, Practical Surface Analysis by Auger and X-ray Photoelectron Spectroscopy, eds., D. Briggs and M.P. Seah, (Wiley, New York, 1983) ch. 4.

[4] P.H. Van Cittert, Z. Phys. 69 (1931) 298.

[5] R.G. Downing, R.F. Fleming, J.K. Langland, and D.H. Vincent, Nucl. Instr. and Meth. 218 (1983) 47.

[6] D.N. Poenaru and N. Vilcov, Measurement of Nuclear Radiation with Semiconductor Detectors (Chemical Publishing Co., New York, 1969) ch. 3.

[7] Radiation Detection, Measurement, Analysis, EG\&G Ortec (1984).

[8] N. Bohr, Phil. Mag. 30 (1915) 581.

[9] E. Friedland and J.M. Lombaard, Nucl. Instr. and Meth. 163 (1979) 523.

[10] P. Sigmund and K.B. Winterbon, Nucl. Instr, and Meth. 119 (1974) 541.

[11] P. Belery, T. Delbar, and G. Gregoire, Nucl. Instr. and Meth. 179 (1981) 1.

[12] G. Spahn and K.O. Groeneveld, Nucl. Instr. and Meth. $123(1975) 425$.

[13] W.M. McKeeman, Commun. ACM 5 (1962) 604.

[14] H.H. Andersen and J.F. Ziegler, The Stopping and Ranges of Ions in Matter. vol. 3 (Pergamon Press, New York, 1977).

[15] D.W.O. Rogers, Nucl. Instr. and Meth. 127 (1975) 253.

[16] G.K. Wertheim, J. Electron Spectros. Relat. Phenom. 6 (1975) 239

[17] Sample courtesy of James R. Ehrstein, NBS, and RBS Measurement courtesy of Charles Evans and Associates.

[18] Sample courtesy of Daniel Oblas of GTE, Waltham Mass.

[19] W.P. Elderton, Frequency Curves and Correlation (Harren Press, Washington DC, 1953) ch. 4.

[20] K.B. Winterbon, lon Implantation Range and Energy Deposition Distributions, vol. 2 (IFI/Plenum Press, New York, 1975).

[21] J.P. Biersack and L.G. Haggmark, Nucl. Instr. and Meth. $174(1980) 257$. 